with positive results for $O$. volvulus on skin analysis $(P=0.023)$.

The authors conclude that the causes for head nodding might be multifactorial. The absence of $O$. volvulus in the patients' cerebrospinal fluid despite the link between positive skin snips and lesions on MRI deserves further investigation.

Original article Winkler AS et al. (2008) The head nodding syndrome-clinical classification and possible causes. Epilepsia [doi:10.1111/j.1528-1167.2008.01671.x]

\section{Cerebral or meningeal vasodilatation does not occur during migraine headaches}

On the basis of the assumption that vasodilatation of intracranial blood vessels is part of the pathologic mechanism underlying migraine headaches, many current antimigraine treatments include agents that constrict these vessels. Such vasoconstrictors are, however, contraindicated in some patients, particularly those with vascular disease. Recent findings by Schoonman and colleagues in fact argue against a prominent role for vasodilatation in the etiology of migraine headaches.

The Dutch study recruited 32 patients (age 18-55 years) who experienced frequent migraines. Nitroglycerine, which reliably induces migraine headaches in susceptible individuals, was intravenously administered to 27 patients $(0.5 \mu \mathrm{g} / \mathrm{kg}$ per min for $20 \mathrm{~min}), 20$ of whom experienced a migraine $1.5-5.5 \mathrm{~h}$ after infusion. None of the 5 patients who received placebo infusions developed a migraine. Intracranial vasodilatation and blood flow was assessed by use of $3 \mathrm{~T}$ magnetic resonance angiography at baseline, during infusion of nitroglycerine or placebo, and during a migraine attack (or $6 \mathrm{~h}$ after infusion if no migraine had occurred). An increase in bloodvessel diameter (6.7-30.3\%) was observed during nitroglycerine infusion; during migraine, however, blood-vessel diameter and blood flow were not significantly different to baseline measurements, nor was there a difference between headache and non-headache sides of the brain in the 18 patients who experienced unilateral headache.

These findings suggest that intracranial vasodilatation does not have a major causative role in migraine headaches. Consequently, vasoconstrictor action might not be an essential requirement for migraine treatments developed in the future.

Original article Schoonman GG et al. (2008) Migraine headache is not associated with cerebral or meningeal vasodilatation - a $3 \mathrm{~T}$ magnetic resonance angiography study. Brain [doi:10.1093/brain/awn094]

\section{Cortical signals can control movement of a prosthetic arm}

Brain-machine interfaces can control simulated actions in a virtual environment, but the capability of these interfaces in a physical setting has not yet been demonstrated. Velliste et al. now report that monkeys can perform a continuous feeding task by use of a brainmachine interface in which cortical signals control a human-like prosthetic arm.

Two Macaca mulatta monkeys were implanted with intracortical microelectrode arrays in their primary motor cortices. Following a training period, the animals were placed in a seated position in which their arms were gently restrained, and a prosthetic arm was positioned next to one of their shoulders. The prosthetic arm contained a two-fingered gripper and had multiple degrees of freedom that enabled human-like movement at the shoulder and elbow. A simple algorithm was used to translate the animals' cortical command signals into arm movements.

During a continuous feeding task, the animals each used the prosthetic arm to reach for food, grab it and place the food in their mouth. The delay between cortical spike signals and arm movement was about $150 \mathrm{~ms}$, similar to that in a natural arm, but the duration of the arm's movements was a few seconds longer than natural movement. Both animals were capable of adjusting the arm's movements as they learned the task (e.g. not gripping sticky food too tightly and changing the arm's trajectory to avoid obstacles) and could use the arm for natural gestures (such as licking the gripper fingers).

The authors state that this experiment paves the way for attaining near-natural function in prosthetic devices.

Original article Velliste M et al. (2008) Cortical control of a prosthetic arm for self-feeding. Nature 453: 1098-1101 